\title{
Primary Pleural Synovial Sarcoma Presenting as Small Nodules Hidden in Pleural Effusion
}

\author{
Kai Lin ${ }^{1,2}$, Biyun Yu³ and Chuan Shao ${ }^{3-5 *}$ \\ ${ }^{1}$ Department of Geriatric Medicine, Ningbo Medical Center Lihuili Hospital, China \\ ${ }^{2}$ Department of General Practice, Ningbo Medical Center Lihuili Hospital, China \\ ${ }^{3}$ Department of Pulmonary and Critical Care Medicine, Ningbo Medical Center Lihuili Hospital, China \\ ${ }^{4}$ Department of Special Procurement Ward, The First Affiliated Hospital of Soochow University, China \\ ${ }^{5}$ Department of General Practice, The First Affiliated Hospital of Soochow University, China
}

*Corresponding author: Chuan Shao, Department of Pulmonary and Critical Care Medicine, Ningbo Medical Center Lihuili Hospital, Ningbo, China and Department of Special Procurement Ward, The First Affiliated Hospital of Soochow University, Suzhou, China and Department of General Practice, The First Affiliated Hospital of Soochow University, Suzhou, Address: No.1111 Jiangnan road, Ningbo Medical Center Lihuili Hospital, Ningbo, 315040, China

\section{ARTICLE INFO}

Received: 幽 July 09, 2020

Published: 幽 July 22, 2020

Citation: Kai Lin, Biyun Yu, Chuan Shao. Primary Pleural Synovial Sarcoma Presenting as Small Nodules Hidden in Pleural Effusion. Biomed J Sci \& Tech Res 29(1)-2020. BJSTR. MS.ID.004742.

Keywords: Pleural Effusion; Primary Pleural Synovial Sarcoma; Thoracoscopy; Diagnosis; Treatment

\section{ABSTRACT}

This is a case report of a middle-aged woman presenting as recurrent hemorrhagic pleural effusion. The patient had only mild symptoms of pain. Pleural effusion examinations showed hemorrhagic exudate and other routine diagnostic methods including bronchoscopy, microbiological examinations, and cytopathology of pleural effusion could not identify the cause. PET-CT showed a metabolically active nodule in the pleural cavity. Over a 3-month period, chest CT showed recurrent pleural effusion with enlarged soft tissue. Several pleural neoplasms and obvious pleural congestion were seen under medical thoracoscopy. However, the specimen exhibited no evidence of malignancy with a medical thoracoscopy biopsy. After multidisciplinary team discussion, the patient underwent surgical thoracoscopy and had comprehensive pleural tumor resection. The pathological results of the surgical specimen showed pleural synovial sarcoma. The patient received chemotherapy with liposomal adriamycin and ifosfamide for five cycles after surgery. Regular follow-ups show that she is doing well.

\section{Introduction}

Pleural effusion is a common condition in respiratory medicine. According to the criteria established by Light, [1] pleural effusion can be divided into exudate and transudate. The causes of exudate are various: most of which can be identified by routine biochemical, microbiological, and pathological examinations. However, nearly $10 \%$ of pleural effusions remain undiagnosed even after medical thoracoscopy [2]. The precise diagnosis of pleural effusion of unknown etiology is usually challenging in clinical practice; it requires multidisciplinary cooperation, especially the assistance of thoracic surgeons and pathologists. Reports of primary pleural synovial sarcoma (PPSS) as the cause of pleural effusion is extremely rare and only a dozen cases have been reported so far. We present a case of hemorrhagic pleural effusion caused by PPSS and outline the step-by-step diagnosis and treatment procedure in this article. Further investigations are needed to determine the optimal treatment of PPSS.

\section{Case Report}

In April 2018, a 47-year-old Chinese woman attended respiratory clinic in our hospital with a 1-month history of pain on the right side of the back. She was a non-smoker and did not have a history of asbestos exposure. Physical examinations revealed decreased vesicular sounds on the lower right lung. Laboratory 
results showed a slight increase of platelet count $(430 \times 109 / \mathrm{L})$, CA125(65.5IU/ml), ESR (70mm/H) and D-Dimer(343ng/ml). Chest contrast enhanced CT showed a small amount of pleural effusion on the right side (Figure 1a). Bronchoscopy showed bronchitis and acid-fast staining of the protected specimen brush was negative. Biopsy of bronchial mucosa showed chronic inflammation.
Thoracocentesis was performed and laboratory tests of pleural effusion showed the appearance was hemorrhagic. The cell counts were $4900 / \mathrm{ul}$ with $70 \%$ mononuclear cells and 30\% multinuclear cells. RBC counts, total protein, LDH and ADA were 324000/ul, $61.8 \mathrm{~g} / \mathrm{L}, 1207 \mathrm{U} / \mathrm{L}$ and $32 \mathrm{U} / \mathrm{L}$, respectively.

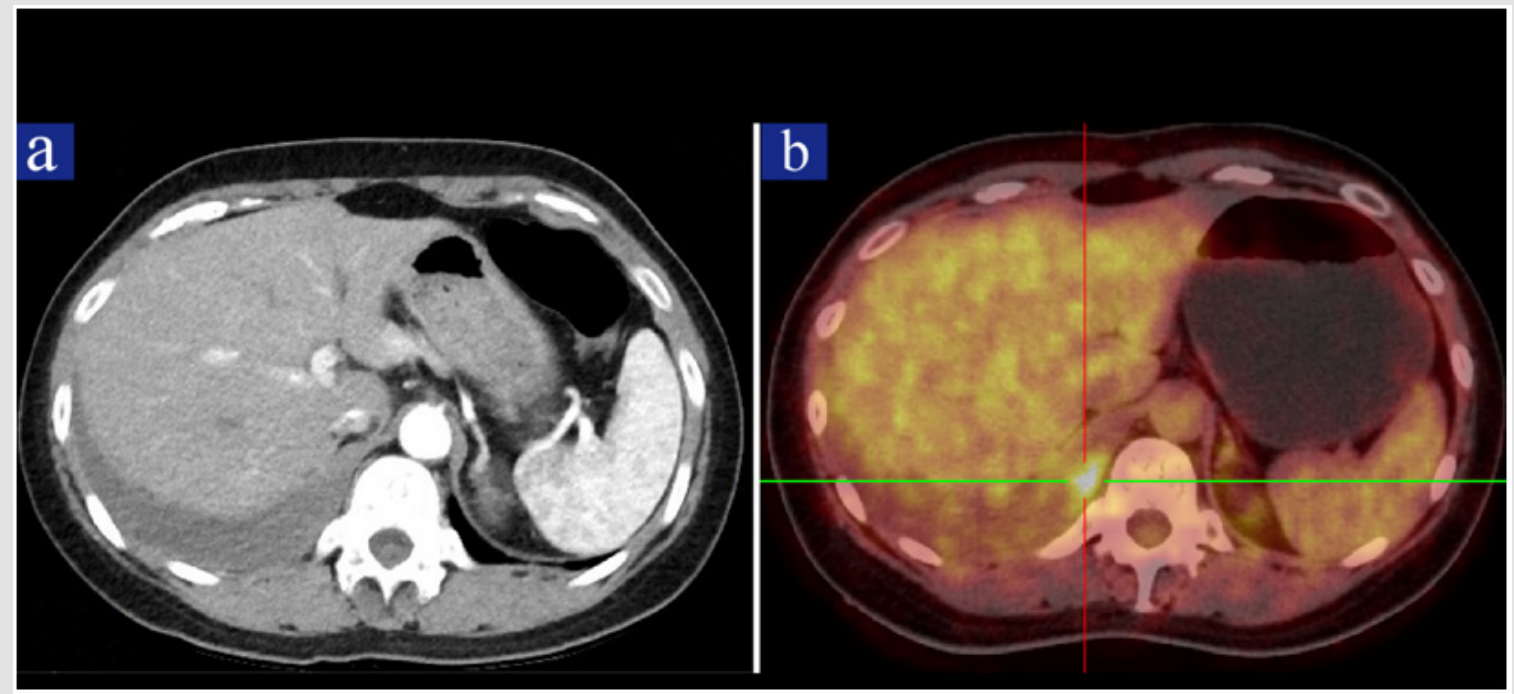

Figure 1: Images of Chest CT and PET-CT scan.

a) Chest contrast-enhanced CT showing a small amount of pleural effusion on the right side.

b) Whole body PET-CT scan showing a metabolically active nodule in the right thoracic cavity.

The level of CEA in pleural effusion was normal. A culture and acid-fast staining of the pleural effusion smear were both negative. Cytopathology of pleural effusion revealed a high number of eosinophils, although, there were some lymphocytes and tissue cells present as well; cancer cells were not found. PET-CT showed a $3.0 \times 1.7 \mathrm{~cm}$ nodule in the right pleural cavity with increased uptake of 18F-FDG and the maximal SUV was 5.7 (Figure 1b). The patient underwent medical thoracoscopy; several pleural neoplasms (Figure 2a), diffuse pleural congestion, and adhesion were discovered. The pleural biopsy revealed hyperplasia and degeneration of fibrous tissue. Comprehensive immunohistochemical (IHC) staining revealed that there was no evidence for diagnosis of any malignancy. Then surgical thoracoscopy was performed and the patient had extensive pleural tumor resection. The pathological findings showed proliferation of atypical spindle cells with mitosis (Figure 2b).

Immunohistochemistry revealed the tumor cells were positive for CD99 (Figure 2c), BCL-2 (Figure 2d), Calponin (Figure 2e), TLE1 (Figure 2f), Calretinin, CD56 and Ki-67(30\%); they were negative for $\mathrm{CK}(\mathrm{pan})$, epithelial membrane antigen(EMA), CD34, cytokeratin(CK)5/6, Wilms Tumor, S-100, SMA, STAT6, Desmin, MyoD1 and Myogenin. The final diagnosis was PPSS. The patient received chemotherapy with liposomal adriamycin and ifosfamide for five cycles after surgery. Regular follow-ups show that she is doing well after chemotherapy. 

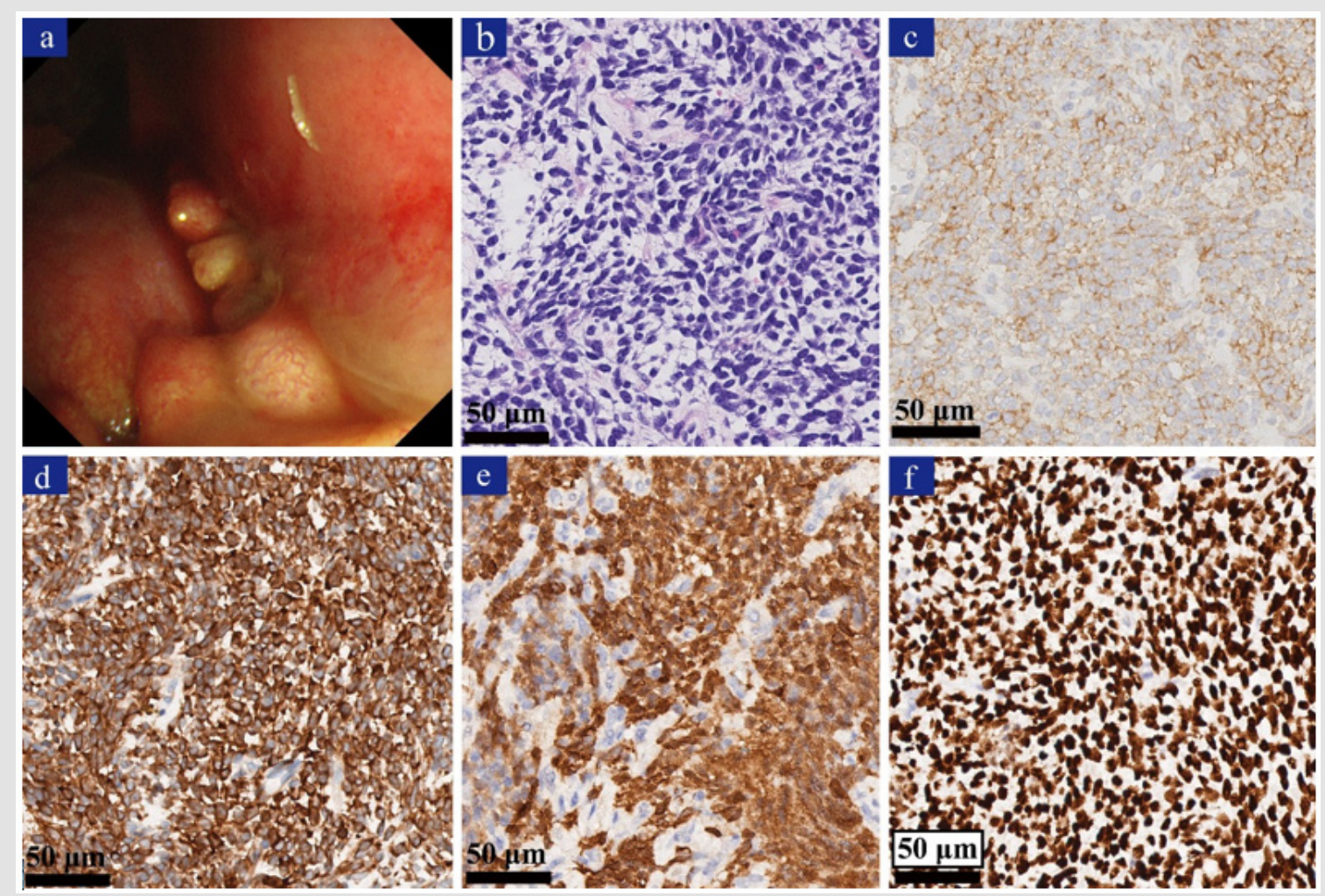

Figure 2: Thoracoscopic and pathological findings of the pleural synovial sarcoma.
a) Medical thoracoscopy showing pleural neoplasms and diffuse pleural congestion.
b) H\&E stain showing proliferation of atypical spindle cells $(x 400)$.
c) Immunohistochemically, the tumor cells showing immunoreactivity for CD99 (c, x400).
d) Immunohistochemically, the tumor cells showing immunoreactivity for BCL-2 (d, x400).
e) Immunohistochemically, the tumor cells showing immunoreactivity for Calponin $(\mathrm{e}, \mathrm{x} 400)$.
f) Immunohistochemically, the tumor cells showing immunoreactivity for TLE1 (f, x400).

\section{Discussion}

Synovial sarcoma is a rare malignancy of mesenchymal origin which mostly affect adolescents and young adults [3]. Although any soft tissue may be involved, it commonly occurs near the joints of the extremities [4]. Synovial sarcoma can be classified as monophasic, biphasic, and poorly differentiated histologically [3]. Primary pleural synovial sarcoma (PPSS) is extremely rare which was first reported in 1996 [5]. The symptoms of PPSS are not distinctive, which may include chest pain, dyspnea and cough, and some patients were asymptomatic [6,7]. The most common radiographic findings are a well-circumscribed mass with effusion [6]. According to previous reports, PPSS are usually large in size without lymph nodes metastasis [4,6-8]. While in the present case, the small lesion was masked by pleural effusion and was difficult to be detected by enhance CT. PET-CT is helpful in finding the tumor and evaluating the regions of involvement.

We believe that favorable outcome may be attributed to early diagnosis in this case. The diagnosis of PPSS is often difficult, as reported by Minoru Yamaki et al. [8] the specimen obtained from CT-guided needle biopsy may be not enough for accurate diagnosis. Medical thoracoscopy was also proved insufficient for diagnosis in our patient-most likely due to the small and superficial of the specimen. Therefore, as in this case, surgical specimens are conducive to diagnosis. The differential diagnosis should include other pleural spindle cell tumors, such as sarcomatous mesothelioma, primary pleural lymphoma, and sarcomatoid carcinoma, etc. Differential diagnosis also includes metastatic disease of pleural from synovial sarcoma elsewhere.

As the differential diagnosis is often challenging, immunohistochemistry is indispensable in confirming the diagnosis. For example, CD99 and B-cell lymphoma 2(BCL-2) are always positive in synovial sarcoma while negative in malignant mesothelioma [8]. Other positive immunohistochemical markers include keratin AE1/AE3, vimentin and actin, and negative markers include CD34, SMA and desmin [7-9].The chromosomal translocation $\mathrm{t}(\mathrm{X} ; 18)(\mathrm{p} 11.2 ; \mathrm{q} 11.2)$, which can be detected by the fluorescence in situ hybridization occurs in more than $95 \%$ of patients, is also an important diagnostic tool $[3,7]$. However, this cytogenetic characteristic is not essential for diagnosis. In the present case, cytogenetic analysis was not performed. 
The best treatment for PPSS remains unclear. A multidisciplinary approach, including surgery, chemotherapy and radiotherapy, can be beneficial according to the limited case reports. Because of its rarity, the evidence-based suggestions for treatment is lacking. Synovial sarcomas are generally considered to be sensitive to certain chemotherapy drugs, such as ifosfamide and doxorubicin [7]. While no guidelines or consensus are available on the role of the chemotherapy on PPSS. Moreover, tyrosine kinase inhibitors pazopanib is approved for the treatment of soft tissue sarcomas and there are clinical trials and case report that suggest it may prolong survival $[3,6]$. Synovial sarcomas are biologically aggressive with a high incidence of recurrence and metastatic potential [3]. Prognosis of PPSS is usually poor with a 5-year survival rates range from $50 \%$ to $75 \%$ [7]. The prognosis may be associated with age, gender, tumor size, site and grade, complete resection or not, and response to chemotherapy, etc. $[3,7]$.

\section{Conflicts of Interest}

The authors have no conflicts of interest to declare.

\section{References}

1. Cornes MP, Chadburn AJ, Thomas C, Darby C, Webster R, et al. (2017) The impact of between analytical platform variability on the classification of pleural effusions into exudate or transudate using Light's criteria. J Clin Pathol 70(7): 607-609.

ISSN: 2574-1241

DOI: $10.26717 /$ BJSTR.2020.29.004742

Chuan Shao. Biomed J Sci \& Tech Res

CC (†) This work is licensed under Creative

Submission Link: https://biomedres.us/submit-manuscript.php
2. Gao BA, Zhou G, Guan L, Zhang LY, Xiang GM (2014) Effectiveness and safety of diagnostic flexi-rigid thoracoscopy in differentiating exudative pleural effusion of unknown etiology: a retrospective study of 215 patients. J Thorac Dis 6(5): 438-443.

3. Silvia Stacchiotti, Brian Andrew Van Tine (2018) Synovial Sarcoma: Current Concepts and Future Perspectives. Journal of Clinical Oncology 36(2): 180-187.

4. Sandeepa HS, Kate AH, Chaudhari P, Chavan V, Patole K, et al. (2013) Primary pleural synovial sarcoma: A rare cause of hemorrhagic pleural effusion in a young adult. J Cancer Res Ther 9(3): 517-519.

5. Gaertner E, Zeren EH, Fleming MV, Colby TV, Travis WD (1996) Biphasic synovial sarcomas arising in the pleural cavity. A clinicopathologic study of five cases. Am J Surg Pathol 20: 36-45.

6. Sugitani A, Asai K, Kojima K, Eguchi Y, Kawaguchi T, et al. (2015) Primary Pleural Synovial Sarcoma Treated with Pazopanib. Intern Med 54: 20512055.

7. Chabowski M, Szymańska-Chabowska A, Janczak D, Dorobisz T, Leśniak M, et al. (2016) A giant pleural poorly differentiated synovial sarcoma (PDSS) in a 64-year-old woman. J Thorac Dis 8(9): E938-41.

8. Minoru Yamaki, Shuji Yonehara, Toshio Noriyuki (2017) Large primary pleural synovial sarcoma with severe dyspnea: a case report. Surgical Case Reports 3: 29.

9. Braham E, Aloui S, Aouadi S, Drira I, Kilani T, et al. (2013) Synovial sarcoma of the chest wall: a case report and literature review. Ann Transl Med 1: 9.

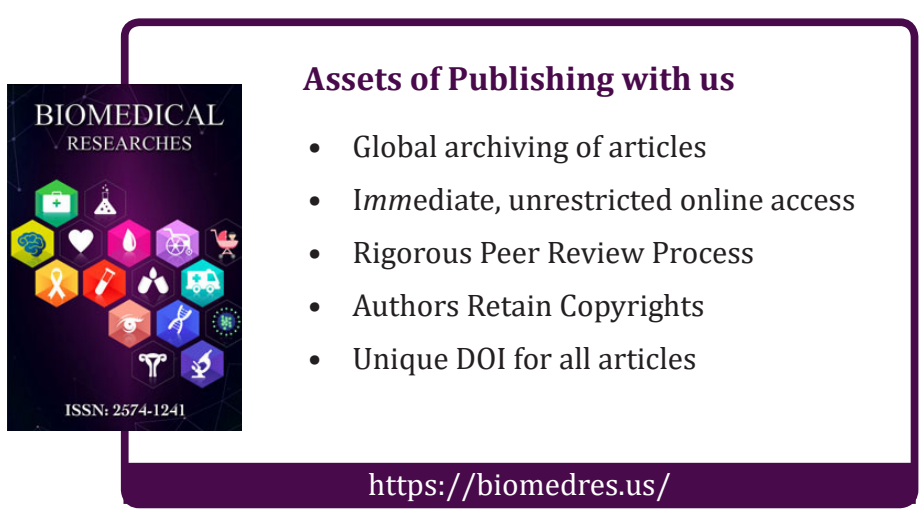

